
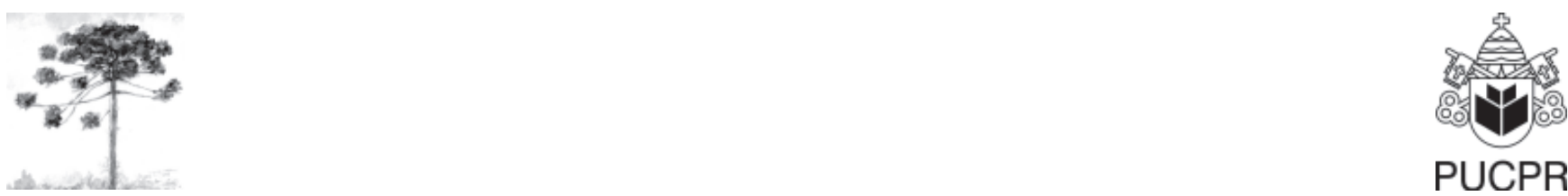

PUCPR

\title{
PARASITISMO POR Balantidium coli EM NUTRIA (Myocastor coypus) NO MUNICÍPIO DE SANTA MARIA, RS - BRASIL
}

\author{
Parasitism by Balantidium coli in nutria (Myocastor coypus) \\ in Santa Maria City, Rio Grande do Sul State, Brazil
}

\author{
Aleksandro Schafer da Silva ${ }^{[1]}$, Régis Adriel Zanette ${ }^{[2]}$, Silvia Gonzalez Monteiro ${ }^{[3]}$ \\ ${ }^{[1]}$ Programa de Pós-Graduação em Medicina Veterinária da Universidade Federal de Santa Maria (UFSM), Santa Maria, RS \\ - Brasil. \\ ${ }^{[2]}$ Programa de Pós-Graduação em Ciências Veterinárias da Universidade Federal do Rio Grande do Sul (UFRGS), Porto \\ Alegre, RS - Brasil. \\ ${ }^{[3]}$ Departamento de Microbiologia e Parasitologia, Centro de Ciências da Saúde da Universidade Federal de Santa Maria \\ (UFSM), Santa Maria, RS - Brasil, e-mail: sgmonteiro@uol.com.br
}

\section{Resumo}

Nutria (Myocastor coypus: Rodentia; Myocastoridae) é um roedor de natureza herbívora natural da região sul da América Latina. A contaminação por protozoários gastrintestinais em vertebrados pode ocorrer através da ingestão de cistos ou oocistos esporulados, os quais podem estar presentes nas fezes, pastagens, água e nos alimentos. O Balantidium é um protozoário ciliado que tem os suínos como hospedeiro natural, porém também é relatado em outros animais. Este estudo teve o objetivo de relatar o parasitismo por Balantidium coli em nutria no Brasil. Foram colhidas e analisadas fezes de duas nutrias, fêmeas, com três meses de idade provenientes de um criadouro conservacionista do município de Santa Maria, RS. As amostras foram mantidas resfriadas e processadas pelo método de centrífugo flutuação com sulfato de zinco. Observou-se no exame parasitológico de fezes uma infecção moderada por cistos de B. coli nos animais, o que leva a concluir que $M$. coypus é hospedeiro desse protozoário. Este estudo é o primeiro registro de parasitismo em nutria no Brasil, possivelmente por causa da restrição de pesquisas envolvendo fauna silvestre.

Palavras-chave: Protozoário; Animal silvestre; Zoonose.

\section{Abstract}

Nutria (Myocastor coypus: Rodentia; Myocastoridae) is a natural herbivorous rodent from Latin America Southern. The contamination by gastrointestinal protozoa in vertebrates can 
occur through ingestion of cysts or sporulated oocysts, which may be present in feces, pasture, water and food. The Balantidium $s p$. is a ciliated protozoan with pigs as natural host, but is also reported in other animals. This study aimed to report the parasitism by Balantidium coli in nutria (Myocastor coypus) in Brazil. Fecal samples from two three-month-old female nutrias from a conservationist breeding center in the municipality of Santa Maria - RS were analyzed. The samples were kept refrigerated and were analyzed by the centrifugal-flotation method with zinc sulfate. The parasitological exam showed moderate infection by cysts of $\mathrm{B}$. coli in the animals, implicating M. coypus as host of this protozoan. This study is the first record of parasitism in nutria in Brazil, possibly due to the restriction of research involving wildlife.

Keywords: Protozoan; Wildlife; Zoonosis.

Nutria (Myocastor coypus: Rodentia; Myocastoridae) é um roedor de natureza herbívora natural da região sul da América Latina. Este animal possui taxa de reprodução relativamente alta, tolerância a variações climáticas e hidrológicas, além de capacidade de adaptação e de dispersão elevada, o que faz com que sua espécie tenha ampla distribuição geográfica (1). Em nutrias é relatado o parasitismo pelas espécies Graphidioides myocastoris sp., Trichuris myocastoris, Dipetalonema sp., Hippocrepis myocastoris sp., Rodentolepis sp. e Giardia sp. $(2,3)$.

O Balantidium é um protozoário ciliado que apresenta distribuição geográfica cosmopolita, sob variadas condições ecológicas, sendo raramente observado em humanos $(4,5)$. Os conhecimentos epidemiológicos sobre esse parasito são escassos e, em vista disso, as recomendações para a prevenção são as mesmas que as usadas para outras parasitoses de disseminação fecal $(5,6)$.

Este ciliado tem os suínos como hospedeiro natural, porém pode ser encontrado no intestino grosso de humanos e outros animais. $\mathrm{O}$ parasito causa a balantidíase que pode apresentar quadros clínicos assintomáticos, crônicos ou agudos com diarreia ou disenteria. O diagnóstico da enfermidade é feito através da visualização de trofozoítos e cistos no exame de fezes ou no tecido coletado durante a endoscopia (4-7).

Conforme a literatura (8), hospedeiros de protozoários das espécies B. coli, Giardia sp. e Entamoeba histolytica são potenciais agentes zoonóticos. Baseado nesses dados julgou-se oportuno relatar o parasitismo por Balantidium coli em nutria (M. coypus) no Brasil.

Para o estudo, foram colhidas fezes de duas nutrias, fêmeas, com três meses de idade provenientes de um criadouro conservacionista do município de Santa Maria, RS, onde estavam alojadas em gaiolas há 25 dias após serem recolhidas pelo IBAMA de uma propriedade rural do município.
As amostras de fezes foram mantidas resfriadas $\left(12^{\circ} \mathrm{C}\right)$ até serem processadas pelo método de centrífugo flutuação com sulfato de zinco (9). A avaliação da carga parasitária foi baseada na classificação de Pinto et al. (10), sendo considerado infecção leve de 1-100 cistos, moderada de 101-300 cistos e elevada quando mais de 301 cistos/lâmina.

Nas amostras analisadas, observou-se presença de cistos nas fezes, identificadas como pertencentes ao gênero Balantidium. Não se observou sinal clínico de parasitose (diarreia e pelagem ouriçada) apesar dos animais apresentarem uma infecção moderada. O parasitismo pelo protozoário já foi relatada em Hydrochaeris hydrochaeris e Agouti paca na América Latina $(11,12)$, mamíferos esses da mesma ordem das nutrias do presente estudo. No Japão, em estudo de prevalência de $B$. coli em roedores das espécies Hystrix cristata e Dolichotis patagona não se mostrou positivo para o protozoário (13). Já em infecção experimental por B. coli em ratos, pesquisadores observaram $75 \%$ de positividade, com eliminação de cistos nas fezes (14).

A literatura nos últimos anos tem registrado estudos de prevalência de $B$. coli em animais silvestres, sendo relatado $84 \%$ de positividade para chimpanzé (Pan troglodytes), 9\% para macacos (Macacafuscata), 100\% para ursos (Sus scrofa) e $38 \%$ para javalis (Sus scrofa scrofa) $(7,13,15$, 16). O ciliado observado neste estudo trata-se do primeiro registro de parasitismo em $M$. coypus no Brasil, possivelmente por causa da restrição de pesquisas envolvendo fauna silvestre brasileira.

Em pesquisa desenvolvida em granjas no Brasil (17), autores observaram B. coli em amostras de suínos de todas as faixas etárias, sendo 3, 1 a 35,0\% dos leitões e 37,0 a $52,0 \%$ das matrizes. Em suínos confinados nos EUA, a literatura reporta valores de 5,0 a 14,0\% em leitões e 18,6\% nos reprodutores (18). São poucos os dados disponíveis acerca deste agente, 
pois este protozoário é considerado um comensal do trato intestinal dos suínos agindo somente como invasor secundário na ocorrência de lesões locais, porém é importante lembrar do seu potencial patogênico para a espécie humana, sendo associado a quadros de disenteria no homem (19). Assim como os suínos, as nutrias parasitadas podem ser uma fonte de contaminação para outros animais silvestres e domésticos ou para o homem, já que esse protozoário apresenta pouca especificidade de hospedeiros.

A balantidíase em suínos domésticos geralmenteéum problema associado ao confinamento, mas pouco se conhece sobre esse parasita em animais silvestres (16). Neste estudo verificou-se que $M$. coypus é hospedeiro do protozoário B. coli.

\section{REFERÊNCIA}

1. Bolkoyic ML, Ramadori D. Manejo de fauna silvestre en la argentina. programas de uso sustentable. dirección de fauna silvestre, secretaría de ambiente y desarrollo sustentable, Buenos Aires: Dirección de Fauna Silvestre; 2006.

2. Barbero BB, Cabello C, Kinoed JE. Helminto fauna de Chile. V. Nuevos parasitos del coipo, Myocastor coypus. Boletim Chileno de Parasitologia. 1979;34:26-31.

3. Dunalp GD, Thies ML. Giardia sp. in Beaver (Castor canadensis) and Nutria (Myocastor coypus) form East Texas. The Journal of Parasitology. 2002;88(6):1254-8.

4. Levine ND. Protozoan parasites of domestic animals and of man. 2nd ed. Minneapolis: Burgess Publishing Company; 1973.

5. Beaver PC, Jung RC, Cupp EW. Examination of specimens for parasites. 9th ed. Philadelphia: Lea \& Febiger; 1984.

6. Gisler DB, Benson RE, Young RJ. Colony husbandry of research monkeys. Annals of the New York Academy of Sciences. 1960;85:758-68.

7. Flynn RJ. Parasites of laboratory animals. Iowa: University Press Ames; 1973.

8. Martino PE, Stanchi NO. Fur bearing animals and zoonoses. World Animal Review. 1991;72:34-6.

9. Hoffman RP. Diagnóstico de parasitismo veterinário. Porto Alegre: Sulina; 1987.
10. Pinto RM, Vicente JJ, Noronha D, Gonçalves L, Gomes DC. Helminth parasites of conventionally maintained laboratory mice. Memórias do Instituto Oswaldo Cruz. 1994;89(1):33-40.

11. Matamoros Y, Velaìzquez J, Pashov B. Intestinal parasites of Agouti paca (Rodentia: Dasyproctidae) in Costa Rica. Revista de Biologia Tropical. 1991;39(1):173-6.

12. Moulton JE, Heuschele WP, Sheridan BW. Balantidiasis in the capybara. Cornell Veterinarian. 1961;51:350-8.

13. Nakauchi K. The prevalence of Balantidium coli infection in fifty-six mammalian species. Journal of Veterinary Medical Science. 1999;61(1):63-5.

14. Karapetian AE, Isaakian ZS, Zavgorodniaia AM. Importance of cellular immunity factors in the pathogenesis of experimental balantidiasis. Parazitologiia. 1978;12(4):323-6.

15. Nakauchi K, Nakajima H, Sakakibara I. Detection of Balantidium coli from evacuated feces in cynomolgus monkeys (Macaca fascicularis). The Japanese Journal of Veterinary Science. 1990;52:1323-4.

16. Mundim MJS, Mundim AV, Santos ALQ, Cabral DD, Faria ESM, Moraes FM. Helmintos e protozoários em fezes de javalis (Sus scrofa scrofa) criados em cativeiro. Arq Bras Med Vet Zootec. 2004;56(6):792-5.

17. Nishi SM, Gennari MNTS, Lisboa A, Silvestrim L, Caproni JR, Umehara O. Parasitas intestinais em suínos confinados nos estados de São Paulo e Minas Gerais. Arq Instit Biol. 2000;67(2):199-203.

18. Morris RG, Jordan HE, Luce WG, Coburn TC, Maxwell CV. Prevalence of gastrointestinal parasitism in Oklahoma swine. American Journal of Veterinary Research. 1984;45(11):2421-3.

19. Rey L. Parasitologia. Rio de Janeiro: Guanabara Koogan; 1991.

Recebido: 08/04/2007

Received: 04/08/2007

Aprovado: 16/06/2007

Approved: 06/16/2007 